\title{
La diáspora en imprenta. Actores, tramas y espacios del libro judío en Buenos Aires, 1910-1960
}

\author{
Alejandro Dujovne
}

Antropología Social

CONICET - Instituto de Desarrollo Económico y Social (IDES). E-mail: aledujovne@gmail.com

\begin{abstract}
Resumen
El artículo analiza desde un punto de vista social y material la producción y circulación de libros en la vida judía de Buenos Aires, como un aspecto clave de su historia cultural. El texto identifica y explora el papel desplegado por librerías, bibliotecas, centros culturales, ferias de libros, editoriales e imprentas en la configuración del mundo del libro judío de Buenos Aires entre 1910 y 1960. A través de la localización de estos actores en un plano de la ciudad de Buenos Aires, se estudia y propone una hipótesis para pensar la relación entre vida social y cultural y espacio urbano.
\end{abstract}

Palabras clave: Historia cultural argentina, Historia del libro, Historia judía.

The diaspora in press. Actors, frames and spaces of Jewish books in Buenos Aires, 1910-1960.

\begin{abstract}
This article analyzes from a social and material perspective the production and circulation of books in the Jewish life in Buenos Aires, as a key aspect of its cultural history. The text identifies and explores the rol undertaken by bookstores, libraries, cultural centers, book fairs, publishing houses and printing presses in the shaping of the Jewish book world of Buenos Aires between 1910 and 1960. Through the localization of these actors in a map of the city of Buenos Aires, it studies and proposes an hypothesis to explore the relationship between social and cultural life and urban space.
\end{abstract}

Keywords: Argentine cultura history, History of the book, Jewish history.

La tarde del viernes 10 de enero, el tío Petacovsky estaba, como siempre, sentado junto a sus libros, tomando mate. (...) A eso de las cinco y media, un grupo de jóvenes bien vestidos hizo irrupción en la acera del boliche, vitoreando a la patria. (...) Uno del grupo, que divisó el rostro amedrentado del tío Petacovsky, llamó la atención de todos sobre el boliche, y los mozos detuviéronse frente al escaparate. -¡Libros maximalistas! -señaló a gritos el más próximo-.jLibros maximalistas! (...)

Samuel Glusberg, "Mate amargo" (La Levita Gris: Cuentos judíos de ambiente porteño, 1924)

Si no fuera porque cuando paseas por Corrientes enloqueces libreros a granel, juf!, judíos harapientos, dueños de los libros hermanos de la dicha.

Washington Cucurto, "Día tras día, un trío de mujeres"

(La máquina de hacer paraguayitos, 2006)
Durante la mayor parte del siglo veinte los libros desempeñaron un papel decisivo en la recreación de la cultura judía argentina, en la que Buenos Aires tuvo un rol central. Mediados por la existencia de un dinámico espacio social de actores culturales, los libros posibilitaron y modelaron formas específicas de circulación y apropiación de las ideas acerca del judaísmo, muchas veces antagónicas, conectando espacios distantes y lenguas distintas. En este sentido, la indagación histórica del mundo social y cultural tras los libros nos reenvía a una serie de combates políticos, culturales e idiomáticos entre diversas corrientes ideológicas judías; coloca a la Ciudad de Buenos Aires dentro de un dinámico sistema transnacional de circulación de obras, revelando su rol cambiante a lo largo del siglo; y nos presenta un abanico de agentes culturales que al construir y ofrecer un repertorio de obras de temática judía en diversos idiomas de acuerdo a sus intereses y posiciones, les otorgaron sentidos específicos.

¿Qué actores modelaron el espacio del "libro judío" en Buenos Aires?, ¿qué intereses y valores los condujeron a invertir en esta clase de obras?, ¿cómo circulaban los libros?, ¿en qué sectores de la ciudad se hallaban

Recibido 18-11-2012. Recibido con correcciones 26-03-2013. Aceptado 25-06-2013. 
situados las imprentas, librerías, bibliotecas y editoriales?, ¿existió algún tipo de relación entre esta clase de obras y el emplazamiento de estas instancias?, y si en efecto existió, ¿qué pone de manifiesto? Este artículo se propone avanzar sobre estas preguntas a través del estudio de un conjunto de actores, espacios urbanos, tramas sociales y medios materiales que configuraron el espacio del "libro judío" de Buenos Aires, tanto en idish como en castellano, entre 1910 y 1950. Dimensiones sociales y materiales susceptibles de análisis socio-histórico, que condicionaron las elecciones editoriales y los modos de circulación tanto de las obras impresas en Buenos Aires como de las importadas, y que, en tanto dieron forma a parte sustantiva del repertorio cultural judío en el país, resultan claves para comprender la recreación y derrotero de la vida cultural judía en éste a lo largo del período analizado.

En las páginas siguientes nos interesa prestar atención a algunos actores de esta trama cultural, no a todos. La elección deja afuera del análisis al primer eslabón de la cadena de producción del libro, el autor, y al último, el lector, y, deberíamos añadir, también traductores. Abarcarlos hubiera exigido una clase de análisis que excede por mucho los límites y preocupaciones iniciales de este texto. En efecto, nuestro énfasis en las dimensiones material y espacial de la vida cultural judía de Buenos Aires nos demanda suspender el estudio de actores y áreas que, al remitir de manera directa a ámbitos, intereses y prácticas culturales altamente diferenciados donde la materialidad y la espacialidad guardan menos centralidad, nos alejan de nuestros objetivos iniciales. ${ }^{1}$

Por "libro judío" tomamos dos definiciones, una que remite a la clase de sello que lo publica, y la otra a los criterios de clasificación utilizados por los propios actores culturales judíos. Es decir, en primer lugar, consideramos como "libro judío" todo libro orientado principal, aunque no únicamente, al público judío, publicado por un sello cuyo catálogo se encuentre dominado por títulos cuyos tópicos se encuentren asociados significativamente a temas judíos en un sentido amplio. Esto incluye tanto títulos en castellano como en otras lenguas publicados dentro o fuera de Argentina. Va de suyo que esta categoría comprende las obras publicadas en idish o hebreo, pues en estos casos la lengua define de antemano el lector potencial al que se encuentran dirigidas. Por "libro judío" también entendemos toda obra de temática judía y/o todo libro de un autor judío reclamado por el mundo cultural judío como tal, más allá de la clase de editorial que lo hubiese publicado. "Libro judío" y "libro de interés judío" fueron las clasificaciones más habituales utilizadas por cronistas y emprendedores culturales de la

\footnotetext{
1 Para un análisis pormenorizado del espacio editorial judío de Buenos Aires ver Alejandro, 2010, Impresiones del judaísmo. Una sociología histórica de la producción y circulación transnacional del libro en el colectivo social judío de Buenos Aires, 1919-1979, tesis doctoral inédita, UNGS-IDES, Buenos Aires
}

época para referirse a títulos en lenguas diferentes del idish y el hebreo que consideraban como judíos. Desde un punto de vista sociológico esta distinción nos permite, entre otras cosas, identificar matices e incluso lógicas diferenciadas en los modos de circulación y apropiación de las obras. De cualquier manera, dadas sus múltiples derivas, solo vamos a tratar este aspecto en función de las necesidades inmediatas del presente artículo.

La elección de la ciudad y del período abordado no son casuales. Buenos Aires, el centro urbano con la mayor población judía de América Latina, vio desplegarse una intensa vida política y cultural judía que la situó desde temprano en un centro de irradiación regional. El período tomado, extenso en términos historiográficos, busca capturar tanto los primeros años de emergencia de este universo cultural como su expansión y cambios. Su cierre se produce a inicios de la década de 1960 ya que, si bien la intensidad y diversidad de la vida cultural judía se mantendrá bien entrada esta década, será en gran medida, y sobre todo en lo que respecta al idish, una extensión de las empresas e instituciones creadas durante el período estudiado. La elección del libro como expresión de la cultura judía tampoco es casual. A través del rastreo de sus huellas el libro nos ofrece un ángulo privilegiado desde el cual reconstruir el devenir cultural y político judío en la ciudad, identificar ámbitos y agentes culturales centrales en este desarrollo, y, tal como lo hicimos en otro trabajo, ${ }^{2}$ aventurar algunas conjeturas acerca de los vínculos materiales y simbólicos de los judíos de Buenos Aires con los grandes centros urbanos judíos de Europa y Estados Unidos por una parte, así como de América Latina por la otra.

\section{La edición judía en Buenos Aires}

La historia de la edición judía de Buenos Aires se divide en dos espacios claramente diferenciados, la producción en lengua idish y la producción en castellano. Durante el período de entreguerras el mercado editorial idish se nutrió principalmente de la importación de libros de los grandes centros editoriales judíos en el mundo, Vilna, Varsovia y Nueva York. A la par de autores nóveles, firmas poco conocidas y traducciones, de estas ciudades llegaban las obras de los nombres consagrados de la ficción, el ensayo y el estudio académico. Por contraste con la producción de estos centros, la edición idish en Argentina durante esta etapa fue escasa y fragmentaria. Esta asimetría entre la fuerza editorial de las primeras ciudades y la relativa debilidad de Buenos Aires se manifiesta no sólo en el plano de la dirección y peso simbólico de los flujos de circulación, sino en el modo en que ese flujo definía las posibilidades y límites de la edición local. Es decir, la fuerza de los grandes centros restringía los márgenes de acción de las iniciativas editoriales de Buenos Aires a la demanda local, y aun dentro de este mercado sus posibilidades se hallaban condicionadas por

\footnotetext{
2 Idem
} 
la presencia de obras importadas.

Hacia fines de la Segunda Guerra Mundial se inicia una marcada transformación en la producción editorial de los sellos judíos tanto en términos cuantitativos como cualitativos que se extiende por al menos dos décadas. Entre 1944 y 1957 se crean una serie proyectos editoriales que modificaron sustancialmente la oferta local así como la posición relativa de Buenos Aires en el universo editorial en esta lengua. Amén de las posiciones culturales o políticas que sostenía cada uno, los ocho sellos más importantes compartían una misma concepción moderna y secular de la cultura judía. Al igual que en la producción editorial de entreguerras, esta expansión debe verse a la luz de las transformaciones de la escena editorial trasnacional y de las maneras en que Buenos Aires participó de ésta. En estos cambios confluyeron distintos fenómenos. Entre los más generales se cuentan los efectos materiales, políticos y culturales del Holocausto, la existencia previa en Buenos Aires de recursos humanos y de una sólida infraestructura en el ámbito de la palabra impresa idish vinculada al periodismo, y una coyuntura económica favorable a la edición. ${ }^{3}$

Aunque no de la misma magnitud, ni por iguales motivaciones políticas y culturales, ni idénticas circunstancias del mercado trasnacional, el ascenso del nazismo y el Holocausto también propiciaron un cambio sustancial en la edición judía en lengua castellana. La producción editorial del período de entreguerras contrasta notablemente con la expansión y reorientación ideológica iniciada en los albores de la Segunda Guerra Mundial. Entre 1919-1938 se realizaron los primeros ensayos editoriales dedicados exclusivamente a temas judíos en lengua castellana en el país. Estos estuvieron motivados, principalmente, por la preocupación de un puñado de intelectuales y activistas culturales acerca de los efectos culturales de la brecha idiomática que separaba el acervo cultural judío de las nuevas generaciones de judíos argentinos. Así como por su convicción de que el libro sobre temática judía constituía una herramienta clave para la legitimación social y cultural de los judíos en el país.

Esta etapa se caracterizó por el predominio de una postura que podemos denominar liberal-integracionista. ${ }^{4}$ El arco integracionista judío se definió por su voluntad de participar como ciudadanos y como miembros plenos de la nación de residencia, sobre la base de una concepción liberal de la sociedad, la política, la religión y el Estado,

\footnotetext{
3 Para un análisis pormenorizado de las condiciones que favorecieron esta transformación, ver Id. Capítulo 3 "El libro idish en la Argentina de posguerra".

4 Véase E. Mendelsohn, On Modern Jewish Politics, Oxford University Press, Oxford, 1993, Págs. 6-17. Para una aproximación general a la concepción liberal en la comunidad judía argentina ver Leonardo Senkman, "Ser judío en Argentina: las transformaciones de la identidad nacional", en Mendes-Flohr, P., Assis, Y. T. y Senkman, L. (Comps.), Identidades judias, modernidad y globalización, Lilmod, Buenos Aires, 2007, Págs. 403-454.
}

y, en el caso específico del grupo de promotores de las iniciativas editoriales, por una marcada distancia de la religión. El fin de esta etapa inicial coincide con la crisis del consenso liberal, tanto dentro como fuera de la vida judía, y con la concomitante expansión de la visión nacional de corte sionista.

A partir de 1938, pero sobre todo desde los primeros años de la década de 1950 en adelante, surgieron nuevos sellos editoriales que contribuyeron a ampliar y sostener una oferta de títulos y tópicos diferenciados, considerados específicamente judíos, dentro del mercado editorial en castellano, fundamental a través de la traducción. Esta expansión fue acompañada de una mayor diversificación ideológica y temática respecto al período anterior. En efecto, sin desplazar del todo a la concepción liberal integracionista, la nueva oferta se distinguió por la reafirmación del carácter nacional judío a través del sionismo, por la irrupción del Estado de Israel como tema y por la traducción de obras pertenecientes a la tradición religiosa judía.

Las editoriales que participaron de la nueva oferta de títulos fueron de muy variado tipo, pero la singularidad y el tono de la oferta estuvo dada por la producción de cinco sellos que se especializaron en obras de interés judío en castellano, publicando de manera casi excluyente traducciones. Estos sellos tuvieron un rol muy importante en la puesta en circulación de un repertorio diferenciado tanto en Argentina como en los países vecinos.

De cualquier manera, la edición de libros de interés judíos no se agotó en la labor de estos sellos. Por el contrario, el número de actores intracomunitarios, principalmente instituciones y corrientes políticas, interesados en participar del mercado editorial creció al ritmo del desarrollo de fuerzas políticas y culturales que procuraban alcanzar al público judío y no judío de habla castellana, y del crecimiento económico general de la colectividad judía. De igual modo, estos sellos no actuaron aislados del resto del mercado editorial. La propia lengua los colocó dentro de un espacio mayor constituido por un amplio número de empresas editoriales generales, locales y extranjeras, que por razones fundamentalmente comerciales optaron por publicar temas y autores que luego eran considerados "libros de interés judíos" por las actores culturales comunitarios. En otras palabras, los sellos especializados no contaron con el monopolio de la publicación de títulos de autores y temas judíos.

\section{Los libros al alcance de la mano: Librerías y bibliotecas judías en Buenos Aires}

\section{a. Las librerías}

Entre los canales a través de los que el lector judío de Buenos Aires pudo acceder a títulos en lengua idish, y a obras de temática judía en castellano, inglés y alemán, 
las librerías ocupan un lugar clave. En el caso del idish las librerías canalizaban la importación de obras de los grandes polos mundiales de edición que conformaban la principal fuente de provisión de literatura del mercado del libro local. Pero el papel de las librerías no se restringió al idish. En este apartado nos interesa identificar las librerías especializadas en libros y publicaciones periódicas judías tanto en idish y castellano, como también en inglés, alemán, hebreo y, en menor medida, otras lenguas.

Algunas de estas librerías, tales como Kaplansky y Sigal, no sólo vendían sino también editaban sus propios títulos en idish y castellano. La librería-editorial Kaplansky, fundada por Gregorio Kaplansky quien arribó al país proveniente de Vitebsk (actual Bielorrusia) en 1912, publica entre 1928 y 1940 alrededor de 20 títulos en idish de distinto volumen, que comprenden dede fábulas para niños, textos escolares, pequeñas obras de teatro escritas por Marcos [Mordejai] Alpersohn acerca de la vida en las colonias, una novela de Israel Yehoshúa Singer y algunas traducciones como Der kop (La cabeza) del médico darwinista italiano Paolo Mategazza y un libro de poesías de Puschkin. Luego de esos años su pequeño catálogo se incrementó con algunas publicaciones esporádicas.

Por su parte, Sigal será reconocida en la segunda mitad del siglo como el principal sello de obras de orientación y estudio religioso. Si bien el primer tramo de su historia editorial está dominado por títulos más cercanos a la clase de obras humanistas de corte liberal- integracionista que publicaba la Sociedad Hebraica Argetina (SHA), su afianzamiento se produce con la edición de títulos de orientación religiosa. Resulta muy probable que esta elección editorial se haya debido inicialmente a la existencia

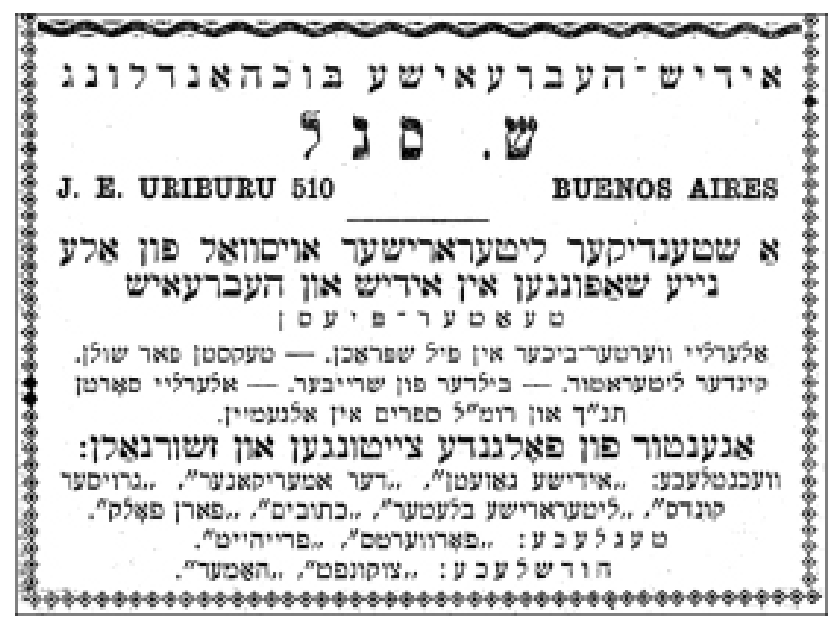

Figura 1. Publicidad de 1928: "Librería idish-hebreo S. Sigal" El anuncio informa que la librería cuenta con las nuevas creaciones literarias en idish y hebreo, identificando los géneros, y que dispone como agente de los siguientes revistas y periódicos importados.

Figure 1. Advertising of 1928: "S. Sigal Yiddish - Hebrew bookstore". The notice informs the library features new creations in Yiddish and Hebrew literature, identifying genres, and which has the following imported magazines and newspapers. de una creciente demanda insatisfecha de textos religiosos básicos en castellano, como por ejemplo una biblia judía. No obstante, no es un dato secundario la afinidad del fundador del sello, Simón, y de su sucesor, su hijo Abraham, con esta clase de obras. Ya desde su lugar de origen, el pueblo de Sfat (Safed), Palestina, Simón adhería al sionismo religioso del partido Mizraji, ${ }^{5}$ en tanto Abraham simpatizaba en el país con el sionismo revisionista, ala derecha del movimiento, que expresaba una oposición a la religión menos tajante que el dominante sionismo socialista. ${ }^{6}$

A la par de las librerías especializadas en, aunque no necesariamente limitadas a, temas judíos, existían otros canales de venta que ampliaban la oferta local. Las publicaciones periódicas constituyeron uno de estos espacios complementarios al ofrecer títulos y publicaciones periódicas importadas. Es el caso de la revista cultural idish de frecuencia mensual Shriftn (Escritos) (1942-1971), cuyo local se situaba en Viamonte 2534, o del semanario en castellano Mundo Israelita que desde al menos 1937 ofrecía a sus lectores, a través de su "Sección librería", dos listas de obras en castellano: "libros de interés judío" (Ilamada inicialmente "Obras y autores judíos"), que en sus primeros años comprendía a obras de temática judía de autores de renombre internacional como Martin Buber, Schalom Asch, y local como Alberto Gerchunoff y a un puñado de obras de temas judíos escritas por nombres reconocidos pero no judíos, tales como Thomas Mann o Jacques Maritain; y otra nómina mucho más extensa de carácter general en la que se encontraban desde Aristóteles hasta Hegel, pasando por Antón Chejov, Rubén Darío y Marcel Proust.

El "Mes del Libro Judío" de la Asociación Mutual Israelita de Argentina (AMIA) ocupa un lugar de relevancia dentro del circuito local de venta de libros. ${ }^{7}$ Desde 1947 y hasta inicios de la década de 1990, el principal centro comunitario judío de Buenos Aires organizó ferias anuales que poco a poco se instalaron como uno de los eventos más relevantes del calendario judío porteño. Más allá de las actividades culturales que ampliaban el interés en la feria, el gran atractivo residía en el número y diversidad de obras ofertadas así como en el precio promocional de éstas (en prácticamente todos los casos contaban con un descuento del 25\%). La amplitud de la oferta y los descuentos ofrecidos funcionaron como un estímulo importante: era una experiencia común que los visitantes aprovechasen cada feria para proveerse de muchos libros

\footnotetext{
5 Acrónimo de las palabras hebreas Merkaz Rujani, "centro religioso", aunque el término también significa "oriental")

6 Entrevista con Ariel Sigal, uno de los actuales dueños de la libreríaeditorial, nieto de Simón e hijo de Abraham.

7 El Mes del Libro Judío no funcionó en 1948, aparentemente por razones políticas e institucionales. La Feria abre un conjunto de dimensiones de análisis fundamentales que hemos tratado en Dujovne, A., 2010, Capítulo 7.
} 


\begin{tabular}{|c|c|c|c|}
\hline Librerías & Años estimados & Ubicación (años estimados) & Otra información \\
\hline $\begin{array}{l}\text { Der Onfang } \\
\text { (El Comienzo) }\end{array}$ & $\begin{array}{l}\text { Probablemente entre } \\
\text { fines de la década de } \\
1900 \text { y } 1914\end{array}$ & Lavalle 2037 & $\begin{array}{l}\text { Librería de Mordejai Stoliar, quien } \\
\text { luego sería uno de los principales } \\
\text { referentes del periodismo idish } \\
\text { como director y redactor de } D i \\
\text { Idishe Tzaitung. Habría sido la } \\
\text { primer librería en traer libros de } \\
\text { la moderna literatura idish al país. }\end{array}$ \\
\hline $\begin{array}{l}\text { Agencia Norte- } \\
\text { Americana Ravitch } \\
\text { \& Berenstein }\end{array}$ & $1910-1920$ & $\begin{array}{l}\text { Bermejo } 467 \\
\text { (actual Jean Jaurés) }\end{array}$ & \\
\hline David Gorodisky & $1910-1940$ & $\begin{array}{l}\text { Av. Córdoba 3358, Dto } 4 \\
\text { Castelli } 395 \text { (entre } 1920 \text { y 1934) } \\
\text { Bustamante } 551 \text { (1934-1940) }\end{array}$ & \\
\hline G. Kaplansky & $1919-1960$ & $\begin{array}{l}\text { Jean Jaures } 467 \text { (década de } \\
\text { 1920) } \\
\text { Av. Corrientes } 2614 \text { (década de } \\
\text { 1930) } \\
\text { Av. Corrientes } 2883 \text { (1940) } \\
\text { Av. Pueyrredón } 451\end{array}$ & $\begin{array}{l}\text { Librería y editorial. La coinciden- } \\
\text { cia entre la primera dirección de } \\
\text { Kaplansky y la de la Agencia Ra- } \\
\text { vitch y Berenstein hace suponer } \\
\text { que Kaplansky dio continuidad a } \\
\text { este negocio refundándolo con } \\
\text { su propio nombre. }\end{array}$ \\
\hline Regalsky y Fain & $\begin{array}{l}\text { 1924-1925 } \\
\text { Seguramente creada } \\
\text { antes y cerrada luego de } \\
\text { esa fecha }\end{array}$ & Ecuador 408 & \\
\hline Sigal & $\begin{array}{l}\text { Desde } 1926 \text { hasta el } \\
\text { presente }\end{array}$ & $\begin{array}{l}\text { Uriburu } 510 \text { (década de 1920) } \\
\text { Av. Corrientes } 2854\end{array}$ & Librería y editorial \\
\hline $\begin{array}{l}\text { Librería idish y cas- } \\
\text { tellano de Shmidt } \\
\text { un Kunpenblat }\end{array}$ & $1935-1945$ & Av. Corrientes 4836 & $\begin{array}{l}\text { La librería cambia de nombre } \\
\text { en determinado momento por } \\
\text { Shmidt un Eijenblat. }\end{array}$ \\
\hline Editorial Israel & $\begin{array}{l}\text { 1938-1952 } \\
\text { Es muy probable que se } \\
\text { haya extendido más allá } \\
\text { de esa fecha }\end{array}$ & $\begin{array}{l}\text { Azcuénaga } 365 \text { (1938) } \\
\text { Sarmiento } 2396 \text { (1938-1945) } \\
\text { Sarmiento } 2198 \\
\text { (1945 en adelante) } \\
\text { (Primeros dos domicilios en el } \\
\text { mismo edificio del semanario } \\
\text { Mundo Israelita) }\end{array}$ & $\begin{array}{l}\text { Durante el primer tiempo importa } \\
\text { libros y publicaciones periódicas } \\
\text { en alemán de Palestina, y luego } \\
\text { en hebreo de Israel y en inglés de } \\
\text { Estados Unidos. A diferencia de la } \\
\text { mayor parte de las otras librerías, } \\
\text { no vende libros en idish. }\end{array}$ \\
\hline $\begin{array}{l}\text { Librería Hebrea } \\
\text { Milberg }\end{array}$ & $\begin{array}{l}\text { Desde } 1947 \text { hasta fines } \\
\text { de la década de } 2000\end{array}$ & Lavalle 2223 & \\
\hline Carlos Hirsch & $\begin{array}{l}\text { 1947-1951 Seguramente } \\
\text { creada antes y cerrada } \\
\text { luego de esa fecha }\end{array}$ & Florida 165 & $\begin{array}{l}\text { La librería se presentaba del si- } \\
\text { guiente modo: "Literatura judía } \\
\text { en idisch, inglés y alemán. Repre- } \\
\text { sentante exclusivo de Schocken } \\
\text { Books" }\end{array}$ \\
\hline Idisch & $\begin{array}{l}\text { La única fecha que } \\
\text { identificamos es } 1951, \\
\text { pero es seguro que fue } \\
\text { creada antes y cerrada } \\
\text { luego de ese año. Es } \\
\text { sucesora de la librería } \\
\text { David Gorodisky }\end{array}$ & Ecuador 637 & De David Gorodisky \\
\hline
\end{tabular}

Tabla 1: Librerías especializadas en temática judía. Fuente: Elaboración propia sobre trabajo de archivo Table 1. Bookstores specializing in Jewish topics. 
o de comprar para regalar. ${ }^{8}$

\section{b. Las bibliotecas durante el período de entreguerras $^{9}$}

Si bien importantes, las librerías no fueron la única fuente de libros para los lectores judíos. Desde las primeras décadas del siglo $\mathrm{XX}$, pero sobre todo durante el período de entreguerras, las bibliotecas proliferaron en todos los barrios porteños donde había alguna presencia judía -y no sólo en aquéllos como Once y Villa Crespo donde esa presencia era más notable- al compás de las necesidades sociales y culturales de los inmigrantes y de la primera generación de argentinos.

En su estudio sobre sociedades barriales y bibliotecas populares, Leandro Gutiérrez y Luis Alberto Romero sitúan a las bibliotecas populares de entreguerras a la par de otros tipos de asociaciones propias de un momento de expansión de la ciudad y de "argentinización" de los inmigrantes (sociedades de fomento, clubes, mutuales, comités de partidos políticos, etc.), aunque las distinguen de éstas por la función cultural y social que cumplían. ${ }^{10}$ Si bien las bibliotecas populares existían desde fines del siglo XIX, los autores indican que su expansión se produjo entre 1920 y 1945.

Al igual que el resto de las bibliotecas públicas creadas durante este período, las judías fueron o bien la base para la fundación de organizaciones de muy diverso tipo o un componente central de las asociaciones culturales, sociales, educativas y políticas luego de que éstas fueron creadas. Además del préstamo de libros, se ofrecían conferencias, cursos, conciertos, reuniones sociales, y fiestas. Así, por ejemplo, en el programa de actos para la segunda quincena de mayo y primera de junio de 1940, la Comisión de Cultura del Club Israelita Argentino Biblioteca "Enrique Heine" ubicado en Avenida Corrientes 1854, informa acerca de dos series de tres conferencias, una del "prestigioso comentarista cinematográfico" León Klimovsky, y otra a cargo del pintor Antonio Berni, así como de un número de conferencias individuales

\footnotetext{
8 Una forma de adquisición de libros publicados fuera del país no despreciable que sería preciso considerar, pero que en este caso escapa a nuestro objetivo más inmediato, parece haber sido la de la compra de obras en el exterior realizada en algún viaje emprendido por el dueño de los libros o por un familiar o conocido, o por el obsequio de visitantes extranjeros. Esta observación se desprende al recorrer las dedicatorias y marcas de los libros en distintas lenguas que se conservan en algunas bibliotecas privadas de Buenos Aires.

9 A diferencia del caso de las librerías, imprentas y editoriales judías de la ciudad estudiadas en este texto, en el de las bibliotecas no hemos avanzado en un relevamiento sistemático y exhaustivo. Su número, variedad y dificultad de rastreo, nos hizo optar por concentrar nuestra atención en algunos aspectos que consideramos relevantes para la descripción y comprensión del universo cultural analizado.

10 Leandro H. Gutiérrez y Luis Alberto Romero, 1995, Sectores populares, cultura y política: Buenos Aires en la entreguerra, Buenos Aires, Sudamericana. Capítulo: "Sociedades barriales, bibliotecas populares y cultura de los sectores populares: Buenos Aires, 1920-1945"
}

entre las que figuran: "Esencia de la democracia" por el Dr. J. M. Velazco Ibarra, ex presidente de la República de Ecuador, "Significado de la Semana de Mayo en la Independencia Argentina" a cargo del Sr. Raúl C. Cabral, y una presentación del profesor Gregorio Berman. ${ }^{11}$ El programa se amplía con dos recitales, el primero a cargo de Isa Kremer y el segundo de Hertz Grosbart. La programación se completaba con dos "revistas orales", actividad en la que confluían comentarios musicales, teatrales, bibliográficos, y cinematográficos, un espacio musical y una sección con información de actualidad de "interés judío". ${ }^{12}$

Nuevamente, a semejanza de lo que tenía lugar en la sociedad general, entre los principales impulsores de la creación de bibliotecas se encontraban las corrientes políticas, en este caso judías. Estas instancias funcionaban, entre otras cosas, como centros de difusión local de obras y autores extranjeros particulares de estas orientaciones ideológicas. Dentro de esta categoría, cabe mencionar, por su temprana creación y por su rol político, a la Biblioteca Rusa, cuya sede fue destruida en 1910 en el marco de la represión obrera. Aun cuando sostuvo un carácter panruso, sus miembros eran mayoritariamente trabajadores e intelectuales judíos, sirviendo de hecho como espacio de confluencia y desarrollo de distintas corrientes políticas judías de izquierda.

En su estudio, Gutiérrez y Romero observan con atención la función integradora ("argentinizadora") de las asociaciones barriales y en particular de las bibliotecas. Éstas habrían constituido un espacio fundamental en el proceso de reconstitución de la cultura de los sectores populares. Pero este énfasis en el carácter integrador, hace que su análisis pierda de vista las bibliotecas étnicas, es decir, las organizadas por los distintos grupos de inmigrantes y de sus descendientes en función de su lengua y cultura, que si bien no contradecían de manera necesaria el proceso de integración, su acción relativizaba o matizaba la linealidad con que estos autores pensaron esta función. Esta nula atención a esta clase de bibliotecas, impide observar la pregnancia que tenían las lenguas de los inmigrantes en la vida social y cultural porteña, así como también, en el caso de quienes hablaban y leían en castellano, de la voluntad de recrear su cultura de origen.

Decimos que las bibliotecas étnicas no necesariamente contradicen la afirmación de Gutiérrez y Romero, sino que la matizan pues, como pudimos apreciar en los casos relevados, los impulsores de las bibliotecas judías parecen haber estado imbuidos del mismo espíritu que los promotores de las bibliotecas populares retratadas en el artículo citado. La composición de la sección

\footnotetext{
11 A pesar de que el apellido se encuentre escrito con una sola " $n$ " final y no con dos, entendemos que se trataba del reputado intelectual y médico psiquiatra Gregorio Bermann.

12 Fuente: Archivo de la Fundación IWO, Buenos Aires. Caja N 62.
} 
castellana de su repertorio revela que participaban de un mismo horizonte de valores culturales que sus pares generales no judías. Un ejemplo entre otros: entre los títulos en idish que figuran en la nómina de libros no devueltos del Centro Cultural Israelita y Biblioteca en Villa Devoto, ubicado en Chivilcoy 2906, del día 27 de octubre de 1935, se encuentran obras de Émile Zola, Friedrich Nietzsche, Fiodor Dostoievski, Henri Barbusse, León Tolstoy, Máximo Gorky, Alejandro Dumas, el premio nobel noruego Knut Hamsun y M. Delly, ${ }^{13}$ a la par de unos pocos autores argentinos como Samuel Eichelbaum, Miguel Cané y Marcelo Peyret. ${ }^{14}$ La mayor parte de estos nombres, que vemos también reiterarse en las distintas listas de obras que ofrecería luego Mundo Israelita en su "Sección librería", eran autores ineludibles para las bibliotecas populares de Buenos Aires. A este respecto, Gutiérrez y Romero explican que su presencia se debe, por una parte, a que "...en el ámbito de las bibliotecas barriales pesó sobre todo la ambición de incorporarse al mundo de la "cultura", es decir al bagaje cultural propio de las elites, acumulado y canonizado."15 Así como, por la otra, al interés por escritores y ensayistas atentos a los problemas sociales desde una perspectiva humanista, cuya obra fuese afín con la sensibilidad integradora de promotores y lectores.

De cualquiera manera, sería erróneo inferir de esto que los lectores judíos optaron exclusivamente por asociarse a bibliotecas judías. Así como participaban de los mismos procesos sociales que el resto de los inmigrantes y primera generación de argentinos, es esperable que de igual modo se hayan asociado alternativa o complementariamente a una biblioteca popular general y participado de sus actividades sociales y culturales. La relevancia analítica de estos espacios físicos y simbólicos compartidos reside en su aptitud para desplazarnos del marco restringido de la circulación de títulos de interés judío en castellano y colocarnos en un sitio con fronteras menos claras que nos permiten una aproximación, aun cuando tangencial, al tránsito de los lectores entre distintas bibliotecas y centro culturales judíos y no judíos, y entre obras y autores universales en castellano y obras y autores judíos (que, durante el período de entreguerras, eran fundamentalmente idish).

Pero el condicionante económico es tan importante en esta historia como el idiomático. En la decisión entre tomar prestado un libro en una biblioteca y adquirirlo en una librería el factor económico tenía un peso considerable. Los inmigrantes judíos se incorporaron a la estructura productiva argentina en las primeras décadas del siglo fundamentalmente como trabajadores. Su

\footnotetext{
13 M. Delly era el seudónimo bajo el cual los hermanos Frédéric Henri Joseph (1876-1949) y Jeanne Marie Petit Jean de la Rosière (18751957) escribieron novelas sentimentales de gran éxito popular entre las décadas de 1920 y 1940

14 Archivo de la Fundación IWO.

15 Leandro Gutiérrez y Luis Alberto Romero, 1995, Pág. 93.
}

ascenso y afianzamiento socio-económico generalizado se produjo de manera más notable desde mediados de la década de 1940 en adelante. ${ }^{16}$ En consecuencia, frente a los múltiples desafíos que el nuevo país les imponía y ante las expectativas que buscaban satisfacer, es comprensible que la biblioteca haya funcionado como un reemplazo o complemento de la compra de obras en librerías. Sobre todo respecto a los libros de literatura idish moderna que hasta el fin de la Segunda Guerra Mundial provenían de los grandes centros editores. A su vez, la inversión de estas condiciones, es decir, una mejora en la posición económica de los judíos y un crecimiento de la oferta editorial local en idish y castellano con la consiguiente baja de precios, nos permite conjeturar que tras 1945 la demanda de títulos en las librerías y por suscripción aumentó, posiblemente, en detrimento del uso de las bibliotecas.

Pese a la importancia de sus funciones como propiciadoras y facilitadoras del acceso del público a libros locales e importados, en castellano e idish, la creación y el desarrollo de las bibliotecas no estuvieron exentos de dificultades. Incluso una biblioteca como la de la Sociedad Hebraica Argentina, creada y sostenida por los cuadros más destacados de la elite intelectual judía de habla castellana, debió enfrentarse a recurrentes problemas. ${ }^{17} \mathrm{Si}$ bien esta biblioteca se presentaba el año de su creación, 1928, como "la única que aspira a ser distinta" por contraste con las numerosas bibliotecas idish-castellano desplegadas por Buenos Aires cuyos libros "... a fuerza de circular, (...) adquieren ese aspecto pastoso y antihigiénico que tanto choca a los amantes de una presentación bonita."18, su principal problema luego de lograr reunir un número suficiente de títulos fue el escaso uso que los socios de la institución hacían de ella.

En un recuadro del boletín de la SHA del 31 de diciembre de 1929 se anuncia que esta publicación institucional comenzará a hacer difusión de los libros con que cuenta la biblioteca, "lamentablemente olvidada" algo más de un año después de su creación. Es interesante comprobar el hecho de que la mayor parte de los títulos listados fuesen en francés y alemán, y, en menor medida, castellano, inglés e italiano. Si bien es cierto que durante esa época resultaba difícil presentar un número relevante de títulos sobre temas judíos en castellano, pues todavía había muy pocos emprendimientos culturales o comerciales interesados en ellos, promover la lectura poniendo en primer plano libros en lenguas conocidas por pocos no

\footnotetext{
16 Al respecto ver Eugene Sofer, 1982, Pág. 128. Acerca de los cambios en el ingreso de los trabajadores durante el período ver Pablo Gerchunoff y Horacio Aguirre, 2006

17 La biblioteca de la SHA se ubica entre las principales bibliotecas judías de la ciudad a lo largo del período estudiado y más allá de él. A la par de ésta, cabría mencionar otras cuya importancia amerita su estudio: la del IWO, la de Congregación Israelita de la República Argentina, las pertecientes a la sociedad B'nai B'rith, etc. 
parecía ser el mejor método para atraer público.

En este apartado hemos situado al desarrollo de bibliotecas públicas judías en el marco de un fenómeno más extendido de expansión de bibliotecas populares en Buenos Aires que tuvo un primer momento de apogeo durante el período de entreguerras. Al hacerlo establecimos una conexión entre un proceso y otro. No obstante, pese a lo evidente que a priori pueda resultar esta relación, el análisis e interpretación de las razones culturales, sociales y políticas históricas que impulsaron la creación de bibliotecas en el caso específico judío requiere la formulación de nuevas preguntas que nos permitan observar motivaciones e intereses particulares de este grupo cultural. Si bien un análisis de esta índole excede el alcance de nuestro objetivo, somos conscientes de su relevancia para aproximarnos desde otra arista a los sentidos atribuidos al libro, identificar una serie de disposiciones y sensibilidades culturales singulares de estos inmigrantes judíos y de sus hijos que nos remite al universo cultural del cual provenían, así como, al igual que en el caso del mundo editorial, reconstruir un conjunto de tramas sociales e institucionales específicas.

\section{c. Expansión de la edición y las nuevas formas de circulación}

La expansión de las editoriales judías argentinas en castellano e idish que tuvo lugar en la posguerra reforzó o dio lugar a nuevas formas de circulación ligadas tanto al precio de los libros como a los canales de venta. La diferencia entre los precios de los libros importados y los producidos en el país favorecía el acceso a los segundos. Por otra parte, los libros publicados en el país se diferenciaban de los importados porque encontraban dos vías de circulación complementarias a las bibliotecas, librerías y el Mes del Libro Judío. Por un lado, los libros eran vendidos directamente por la editorial a través de agentes y suscripciones. Por el otro, el carácter institucional de las editoriales en idish más grandes, hacía que los libros se pudiesen adquirir en los locales de las instituciones. De tal manera que la cercanía y la participación en actividades de un partido político, una escuela o una sociedad de coterráneos podía implicar la compra de los títulos publicados por su sello. Más aún, esta adhesión a una de estas instituciones orientaba las suscripciones.

En los márgenes de estas instituciones y canales formales, se hallaba la mayor parte de los escritores idish locales, quienes, por dicha marginalidad, se veían triplemente perjudicados. En primer lugar, la falta de apoyo institucional se traducía en la exigencia de tener que autofinanciar la edición de sus propias obras o de buscar ayuda de familiares o conocidos para poder hacerlo. En segundo término, verse excluidos del dispositivo cultural de jerarquización de la producción literaria que constituían las editoriales más importantes -en tanto sólo se intereseaban por la producción local de modo excepcional y muy selectivo, editando en su mayoría autores idish que residían en los grandes centros judíos-, suponía que de antemano su obra tuviera un menor valor relativo. Por último, y en lo que a este apartado respecta, esta exclusión los colocaba fuera de los canales de distribución desplegados por los sellos. Como resultado de ello, los escritores locales se veían obligados a vender directamente sus obras casa por casa, comercio por comercio.

\section{d. Las imprentas}

Las imprentas constituyen una pieza fundamental en el proceso de producción editorial. Sin ellas, sencillamente, no habría habido libros tal como los conocemos hoy ni el impacto histórico de éstos hubiera sido el mismo. Pero más allá de esta constatación de sentido común, la historia y la sociología del libro han repuesto a este actor en las tramas sociales del proceso editorial demostrando otros aspectos de su incidencia en la producción material y simbólica de los libros. ${ }^{19}$ Considerando esto, pero sabiendo que el extenso programa de investigación que se desprende de estos enfoques sobrepasa los márgenes de este trabajo, en este punto nos interesa recuperar ciertos planos que nos ayuden a completar el cuadro socio-histórico que procuramos trazar. En tal sentido nos interesa comprender cómo se insertaban los talleres gráficos dentro del espacio de producción editorial idish por una parte y castellano por la otra, al tiempo que identificar a los dueños de las imprentas y establecer qué clase de vínculos tenían con la vida política y cultural judía de Buenos Aires.

Para publicar obras en lengua idish se precisaba de imprentas y linotipias que contaran con tipos hebreos, así como con componedores y correctores con competencia en esta lengua. De modo que no cualquier taller gráfico estaba en condiciones de tomar encargos de libros y publicaciones periódicas en idish. Pero por otra parte, aquellas empresas que sí contaban con la infraestructura y los recursos humanos para hacerlo, no se ceñían a ella e imprimían también para el más amplio mercado en castellano. Pese al número de imprentas con capacidad para confeccionar libros en idish, durante el momento de expansión de la edición local en esta lengua el mercado estuvo dominado por un par de ellas, Zlotopioro Hermanos y Julio Kaufman. Dominio que no implicaba que las otras fueran apenas marginales pues el mercado en lengua idish comprendía productos distintos al libro, desde periódicos hasta papelería de oficina y publicidad. A partir de los pies de imprenta de los libros relevados y de anuncios publicitarios, elaboramos un cuadro con las imprentas de Buenos Aires que publicaron al menos un libro en idish.

Las trayectorias de los dueños de las dos principales imprentas ofrecen claves importantes para nuestro análisis. Ambas imprentas tienen un mismo punto de

\footnotetext{
19 Al respecto ver, por ejemplo, la obra de Robert Darnton.
} 
partida. David Zlotopioro, dueño del taller de imprenta junto con su hermano Jacobo, y Julio Kaufman nacieron en dos pequeños pueblos polacos, el primero en 1903 y el segundo un año después ${ }^{20}$. Julio Kaufman llegó al país alrededor de 1920 y comenzó como aprendiz de imprenta hasta que ganó suficiente experiencia para probar suerte por su cuenta. Para ello trajo a sus hermanos y abrió una pequeña empresa familiar con su nombre. Por su parte, David Zlotopioro arribó a Buenos Aires con su esposa desde Varsovia algo más de una década después que Kaufman. Empezó a trabajar como tipógrafo primero y linotipista luego en el diario progresista idish Di Prese. Su hermano se suma a la mismas tareas poco después tras residir un tiempo en Uruguay. Cuando se encontraron en condiciones de hacerlo adquirieron una linotipo que, tras cierto lapso, les permitió dejar el trabajo en el periódico para dedicarse por entero a su propia empresa.

Al igual que muchos de su generación, Julio Kaufman tomó distancia de la religiosidad tradicional de su padre, quien era matarife ritual y había sido fundador de una sinagoga en el barrio del Once, para abrazar el sionismo socialista y en política nacional argentina adherir con entusiasmo al Partido Socialista. En tal sentido su hijo Eduardo recuerda haber visto a Alfredo Palacios y a Alicia Moreau de Justo brindando conferencias en algunas de las tertulias organizadas en su domicilio. Los hermanos Zlotopioro optaron en el inicio por una adhesión distinta, la izquierda no sionista del ICUF. ${ }^{21}$ No obstante, rompen con ésta cuando en 1952 los líderes de este campo ideológico se negaron a reconocer y condenar los procesos llevados cabo contra los escritores judíos en la Unión Soviética. Por otra parte, Eduardo Kaufman y Alberto Zlotopioro, hijos respectivos de Julio y David, recuerdan que sus padres conocían y tenían vínculos con distintos intelectuales y editores judíos.

Los Talleres Gráficos Julio Kaufman llegaron a contar con 10 obreros, algunos de ellos necesariamente judíos puesto que por la clase de títulos publicados se precisaba de operarios calificados con una alta competencia en lengua idish. Julio fue el único dueño de la imprenta hasta 1960, año en que emigra a Israel. Los talleres gráficos cierran definitivamente sus puertas en 1979. Por su parte Zlotopioro Hermanos continua existiendo hasta el presente. Tras la muerte de David y Jacobo fueron los hijos de ambos quienes prosiguieron con la empresa. De acuerdo al actual dueño

20 Para la reconstrucción de las trayectorias de David Zlotopioro y Julio Kaufman nos apoyamos en las entrevistas que realizamos a sus hijos, Alberto Zlotopioro (enero de 2010) y Eduardo Kaufman (abril de 2009).

21 El Idisher Cultur Farband (ICUF) se fundó en Buenos Aires en 1941. EI ICUF fue, y con cambios hoy aun es, una federación de entidades culturales y sociales judías creada como expresión local de la asociación cultural judía del mismo nombre conformada en París en 1937 durante el Congreso de Escritores Antifacistas promovido por la nueva posición de "frentes populares" establecida por la Internacional Comunista en 1935. Desde su mismo origen el ICUF estuvo ligado al Partido Comunista Argentino. de los talleres gráficos, Zlotopioro fue la última imprenta que realizó trabajos en idish, los cuales se llevaron a cabo a finales de la década de 1980. Si bien ambas se destacaron del resto por haber impreso la mayor parte de los libros de las editoriales idish más importantes, también publicaron obras en castellano y revistas y periódicos en ambos idiomas.

Estas trayectorias demuestran que la fase de la impresión dentro del proceso de producción no puede ser concebida, al menos en este contexto, únicamente en términos económicos. En otras palabras, la participación de estas imprentas en la producción editorial y más aún su posición dominante durante la posguerra, no sólo pareciera haberse debido a una razón de costos y a la realización de un trabajo satisfactorio, sino también al modo en que estos pequeños empresarios participaron de las distintas tramas sociales y culturales judías. Efectivamente, estos recorridos revelan experiencias, convicciones y compromisos políticos dentro del campo judío, cierta formación y sensibilidad literaria, así como un arco de relaciones sociales. Por contraste, es muy probable que el peso de las trayectorias y de las relaciones sociales haya tenido un peso menor en el espacio de la edición en castellano frente al criterio económico y de calidad. Ello es así porque, de hecho, las ediciones en castellano no precisaban restringirse a los talleres gráficos que contaran con tipos hebreos y componedores y correctores competentes en idish. De esta suerte, la opción por el castellano al tiempo que diluía el peso de estos vínculos intrajudíos en la elección de la imprenta, ampliaba el mercado de las imprentas posibles. ${ }^{22}$

\section{La espacialidad de la producción y la circulación}

En las citas que abren este artículo, Samuel Glusberg (bajo el nom de plume de Enrique Espinosa) y Washington Cucurto sugieren la existencia de una relación especial entre judíos, libros y cierta geografía urbana de Buenos Aires. ${ }^{23}$ Esta relación tiene, como intentaremos demostrar aquí, una singular importancia para comprender parte de la vida cultural judía en la Argentina.

La producción y circulación de libros guarda una dimensión espacial por antonomasia que, en este caso, se expresa en áreas específicas de la ciudad de Buenos Aires. Así, a partir de un plano confeccionado sobre la base de las nóminas de librerías, editoriales e imprentas, nos interesa identificar y comprender ciertos aspectos de la relación entre espacialidad y producción y circulación

\footnotetext{
22 Así, por ejemplo, la Editorial Israel optó para la mayoría de sus libros por la imprenta López situada en Perú 666 en el barrio de San Telmo, y Candelabro alternó entre imprentas tradicionalmente ligadas a la edición de libros judíos en idish o castellano, como Zlotopioro, y otras no como Macland S.R.L. ubicada en Córdoba 3965 en el Barrio de Palermo.

23 "Mate amargo" narra el asesinato de un librero judío, el "tío Petacovsky" en el barrio del Once a manos de jóvenes patricios durante la Semana Trágica en 1919. Samuel Glusberg escribe el relato bajo el nom de plume deEnrique Espinosa. La levita gris. Cuentos judíos de ambiente porteño, Ed. Babel, 1924.
} 


\begin{tabular}{|c|c|c|}
\hline Nombre de la imprenta & $\begin{array}{l}\text { Período de } \\
\text { funcionamiento }\end{array}$ & Domicilio y período en que permaneció en é \\
\hline $\begin{array}{l}\text { Krasilovsky y Pertzovsky } \\
\text { (Krasilovsky luego sigue solo) }\end{array}$ & 1918-1938 & Valentín Gómez 2700 \\
\hline $\begin{array}{l}\text { Cultura } \\
\text { de Muskat y Saslavsky }\end{array}$ & 1931-1966 & $\begin{array}{l}\text { Tucumán } 3067 \text { (1931-1932) } \\
\text { Sarmiento } 2157 \text { (1934-1966) }\end{array}$ \\
\hline Talleres Gráficos A.J. Weiss & 1935 & Riobamba 562 \\
\hline Julio Kaufman & 1936-1979 & $\begin{array}{l}\text { Junín } 344 \text { (1936-1951) } \\
\text { Corrientes } 1976 \text { (1950-1979) }\end{array}$ \\
\hline $\begin{array}{l}\text { Poliglotn } \\
\text { de M. Perzovsky }\end{array}$ & $1938-1943$ & Corrientes 3114 \\
\hline Talleres Gráficos Heuman & $1946-1954$ & Pasteur 333 \\
\hline Zlotopioro Hnos & 1948-hasta hoy & $\begin{array}{l}\text { Gascón } 1231 \text { (1948-1956) } \\
\text { San Luis } 3149 \text { (1957-1975) } \\
\text { Sarmiento } 3149\end{array}$ \\
\hline $\begin{array}{l}\text { Impresora Baires } \\
\text { de Simón Eidelman }\end{array}$ & 1951-1953 & Cangallo 2981 \\
\hline $\begin{array}{l}\text { Talleres Gráf. Optimus SRL } \\
\text { (probablemente de Krasilovsky) }\end{array}$ & 1950-1967 & Valentín Gómez, 2715-19 \\
\hline $\begin{array}{l}\text { Graficon } \\
\text { de N. Zielony }\end{array}$ & 1951-1959 & $\begin{array}{l}\text { Pasteur } 356 \text { (1951-1956) } \\
\text { Loria } 618 \text { (1959) }\end{array}$ \\
\hline Optimus & 1952 & Valentín Gómez 2715-19 \\
\hline Talleres Gráf. Kalifón SRL & 1954 & Miró 747 \\
\hline $\begin{array}{l}\text { Talleres Gráficos México } \\
\text { de Simón Eidelman }\end{array}$ & 1955 & México 2230 \\
\hline
\end{tabular}

Tabla 2: Imprentas de Buenos Aires que publicaron libros en lengua idish .Fuente: Elaboración propia sobre la base de trabajo de archivo

Table 2. Printers in Buenos Aires who published books in Yiddish.

del libro, así como, por otra parte, explorar en qué medida esta perspectiva nos proporciona un nuevo ángulo desde el cual observar e interpretar algunos fenómenos estudiados en trabajos precedentes. ${ }^{24}$

Uno de los índices para observar el grado de asimilación o de segregación de los inmigrantes y de sus descendientes en el plano de las interacciones sociales son las pautas de inserción residencial. No obstante, como bien ha señalado Fernando Devoto, la concentración geográfica no redunda de manera necesaria en mayor interacción social, ya que contigüidad no es igual a vínculo social. ${ }^{25}$ En todo caso, una estrategia más adecuada para estudiar la relación entre espacialidad e intensidad de las relaciones sociales, pareciera ser el análisis de la relación entre las pautas de asentamiento y la localización de las asociaciones étnicas. En el caso judío esto es particularmente significativo puesto que si bien hubo dos barrios de Buenos Aires con una alta concentración judía, Once y Villa Crespo, el primero de ellos

\footnotetext{
24 Nos referimos a Alejandro Dujovne, Cartografía de las publicaciones periódicas judías de izquierda en Argentina, 1900-1953, Revista del Museo de Antropología 1(1): 121-138, 2008, pp. 121-138, y a los capítulos 2 a 5 de Alejandro Dujovne, 2010, Op.Cit.

25 Fernando Devoto, Historia de la inmigración en la Argentina, Sudamericana, Buenos Aires, 2009, p. 341.
}

fue el que concentró la mayor parte de la vida institucional.

El plano editorial, por su parte, tiene una correspondencia espacial en términos específicos. En su estudio sobre el campo francés de la edición Bourdieu establece una correlación entre grado de posesión de capitales simbólico y económico y ubicación geográfica de las editoriales. ${ }^{26}$ La tendencia identificada en su estudio señala que mientras más capitales reúnan estos sellos más cerca se ubicarán del centro de París, y de modo inverso, mientras menos capitales posean más lejos se encontrarán, llegando incluso a situarse en las provincias y en el extranjero. Con la exposición de este vínculo, Bourdieu sugiere la existencia de una relación entre centralidad en el campo editorial y centralidad geográfica. Si bien el sociólogo francés no se preocupa en extraer de esta constatación sus posibles consecuencias teóricas, se desprende de su observación que la cercanía al centro de la geografía cultural refuerza el prestigio de un sello, al tiempo que, en términos de capital social, la coloca en un espacio de mayor circulación de actores de relieve. Ahora, ¿en qué medida nuestro caso responde a esta idea? Tal vez podamos responder a este interrogante y proponer algunas

26 Pierre Bourdieu, "Una revolución conservadora en la edición" en Intelectuales, política y poder, Eudeba, Buenos Aires, 2000. 


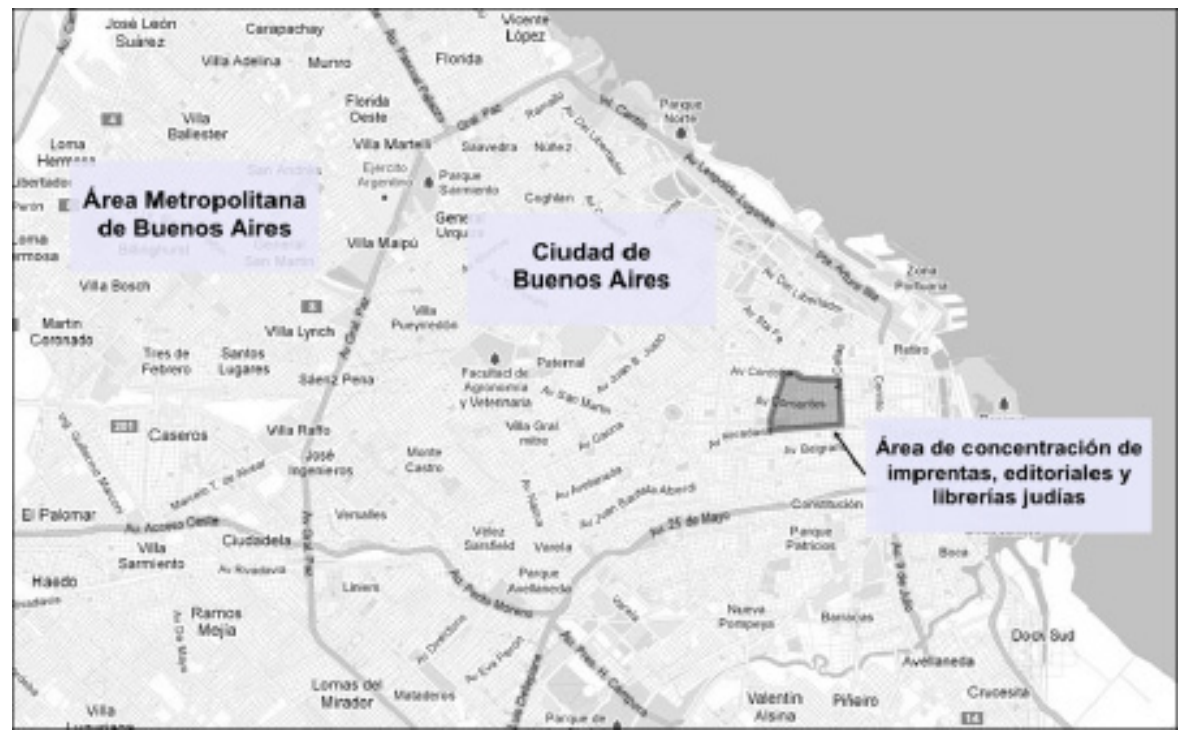

Figura 2. Área de concentración de las imprentas, librerías y editoriales judías en la Ciudad de Buenos Aires, 1910-1980.

Figure 2. Jewish Printers, Bookstores an Editorials concentration area in Buenos Aires, 1910-1980.

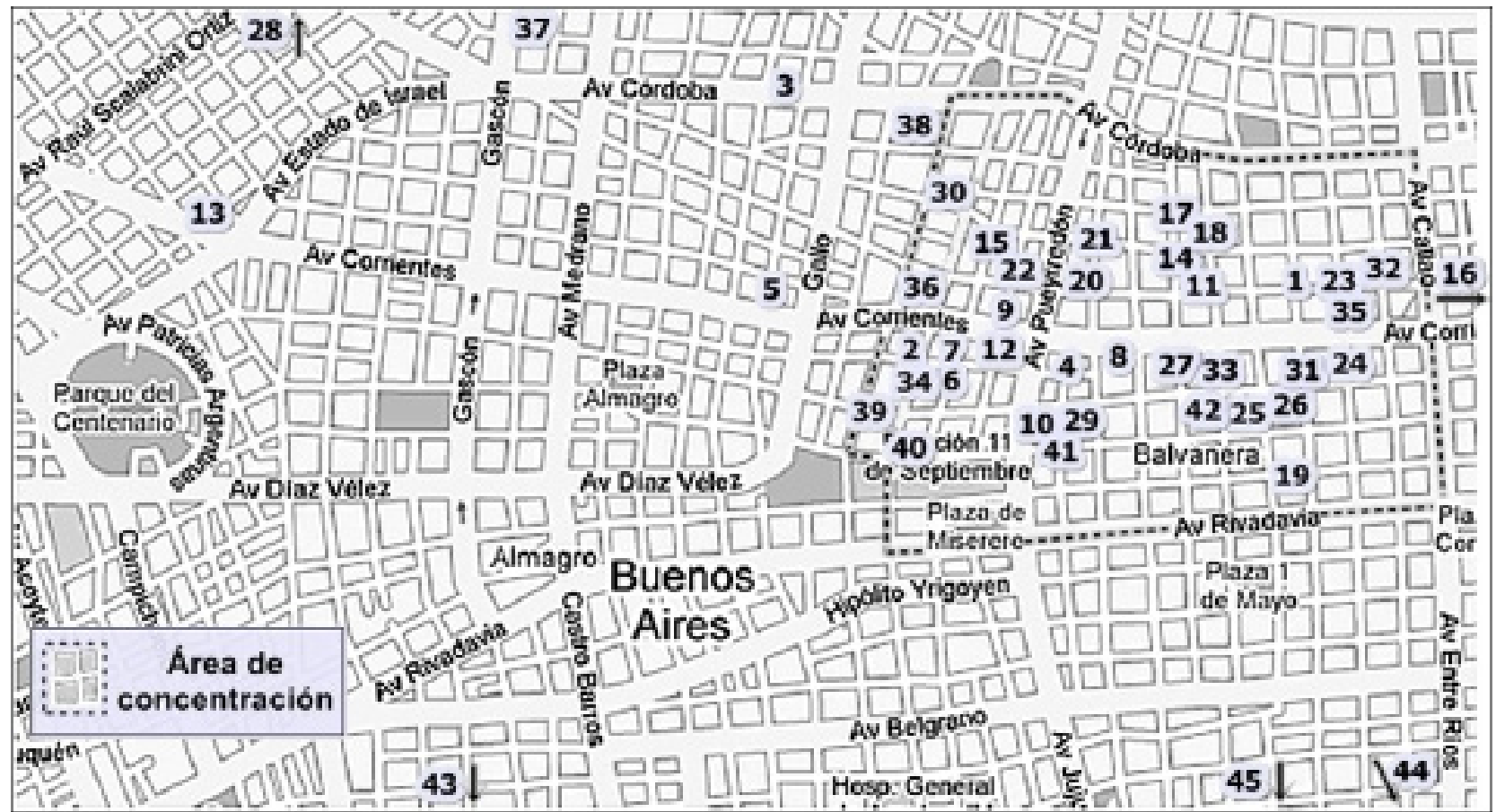

Figura 3. Foco sobre el área de concentración de librerías, editoriales e imprentas, 1910-1980. Fuente: Elaboración propia sobre plano de Google Maps. Referencias (ver abajo)

Figure 3. Zoom on the Jewish Printers, Bookstores an Editorials concentration area in Buenos Aires, 1910-1980. References (see below)

Librerías/Bookstores: 1- Der Onfang, probablemente entre 1910 y 1914, Lavalle 2037; 2 -Agencia Norte-Americana Ravitch \& Berenstein, 19101920, Bermejo 467 (actual Jean Jaurés); 3- David Gorodisky, 1910-1929, Avenida Córdoba 3358, depto 4; 4- David Gorodisky, primera mitad de la década de 1930, Castelli 395; 5- David Gorodisky, 1934-1940, Bustamante 551; 6- Regalsky y Fain, 1924-1925, Ecuador 408; 7- G. Kaplansky, Jean Jaures 467 (1920s); 8- G. Kaplansky, décadas de 1920 y 1930, Av. Corrientes 2614; 9- G. Kaplansky, décadas de 1940 y 1950, Av. Corrientes 2883; 10- G. Kaplansky, década de 1960, Av. Pueyrredón 451; 11- Sigal, 1926- fines de la década de 1920 o principios de la de 1930, Uriburu 510; 12- Sigal, fines de la década de 1920 o principios de la de 1930-hasta el presente, Av. Corrientes 2854;13- Librería idish y castellano de Shmidt un Kunpenblat, y luego Shmidt un Eijenblat 1935-1945, Av. Corrientes 4836; 14- Librería Hebrea Milberg, 1947- hasta el presente, Lavalle 2223; 15- Idisch, 1951 ¿?, Ecuador 637; 16- Carlos Hirsch, 1947-1951, Florida 165; 17- Amia - Mes del Libro Judío, 1947-1982, Pasteur 633; Editoriales/Editorials: 18- IWO / Musterverk, Pasteur 633; 19- Farlag Idish, Bartolomé Mitre 2146; 20- ICUF, Pueyrredón 652; 21- Dos Poylishe Idntum, Pueyrredón 667; 22- Idbuj -Boulonge Sur Mer 671-675; 23-Cultur Congres -Ayacucho 483; 24- Kium -Ayacucho 352; 25- Sociedad Hebraica Argentina - Sarmiento 2233; 26- Israel - Sarmiento 2198; 27- Candelabro - Pasteur 341, 3er piso Of. 1; 28- Acervo Cultural - Nicaragua 4462; Imprentas que publicaron libros en idish/Printers who published books in Yiddish: 29- Krasilovsky y Pertzovsky, 1918-1938 Valentín Gómez 2700; 30- Cultura de Muskat y Zaslavsky - 1931-1932, Tucumán 3067; 31- Cultura de Muskat y Zaslavsky - 1934-1966, Sarmiento 2157; 32- Talleres Gráficos A.J. Weiss, 1935, Riobamba 562; 33- Heuman - 1946-1954, Pasteur 333; 34- Julio Kaufman - 1936-1951, Junín 344; 35- Julio Kaufman - 1950-1979, Corrientes 1976; 36- Poliglotn de M. Perzovsky, 1938-1943, Corrientes 3114; 37- Zlotopioro Hnos - 1948-1956, Gascón 1231; 38- Zlotopioro Hnos - 1957-1975, San Luis 3149; 39- Zlotopioro Hnos - 1975, Sarmiento 3149; 40- Impresora Baires, 1951-1953, Cangallo 2981; 41- Talleres Gráf. Optimus SRL - 1950-1967 Valentín Gómez, 2715-19; 42- Graficon de N. Zielony - 1951-1956, Pasteur 356; 43- Graficon de N. Zielony -1959, Loria 618; 44- Talleres Gráf. Kalifón SRL - 1954, Miró 747; 45- Talleres Gráficos México - 1955 México 2230. 
otras conjeturas al plasmar el mundo judío del libro en un plano de la ciudad.

Al situar en un plano de la ciudad al conjunto de librerías, editoriales e imprentas, colocando en un mismo plano temporal sus traslados de una dirección a otra, descubrimos que la ciudad de la que estamos hablando ya no es el amplio y heterogéneo espacio urbano que tenemos en mente sino una zona mucho más acotada: un barrio, el Once.

Desde una perspectiva económica algunas cuestiones parecen evidentes. Una librería decide el lugar de su emplazamiento en función de la demanda potencial que ese entorno le provee. Esto explicaría entonces tanto la ubicación inicial como la decisión respecto de la nueva ubicación cuando deben u optan por mudarse. Los desplazamientos demuestran que cuando debieron mudarse eligieron hacerlo dentro del mismo radio geográfico. Este es, por ejemplo, el caso de G. Kaplansky, quien realizó sus cuatro movimientos dentro de un radio no mayor a cinco manzanas. Algo similar sucede en el caso de las imprentas. El Once las acercaba a un mundo comercial, profesional y cultural judío (instituciones, redacciones de periódicos, empresas, editoriales, etc.) al cual podían ofrecer sus distintos servicios de impresión. Pues, como lo recuerda Alberto Zlotopioro, las imprentas idish eran requeridas para diversos tipos de impresos (tarjetas personales, de salutación, hojas membretadas, etc.) que iban más allá de las publicaciones periódicas y los libros. Por otro lado, el Once no les impedía que pudiesen también dedicarse a la impresión en castellano, pues este barrio se situaba muy cerca del centro de la ciudad y por ende del corazón profesional y comercial de ésta. Pero lo contrario a ello sí sucedió, así como las editoriales idish dependían de la existencia de estos talleres gráficos para poder publicar, las editoriales judías en castellano no se veían condicionadas por esta oferta y recurrían a imprentas no judías, probablemente más económicas, situadas en otros barrios de la ciudad. Esto revela, como ya adelantamos, que la lengua no sólo funcionaba como un límite cultural y social, sino también en este caso económico y geográfico.

En el caso de las editoriales el beneficio económico no es tan inmediatamente evidente como en el de las librerías y las imprentas. En la medida en que sus ventas no dependen de la mayor o menor circulación de personas por la zona en que se encuentran emplazadas, las editoriales podrían haber prescindido a priori de afincarse en el Once. Tal vez, siempre desde un punto de vista económico, la cercanía con las principales librerías e instituciones centrales de la vida judía, las haya beneficiado.

De todos modos, difícilmente pueda reducirse el análisis al mero cálculo económico. En efecto, no puede despreciarse la intensidad de la interacción social judía en el barrio del Once ni tampoco puede desestimarse el valor simbólico de encontrarse en este barrio. En primer término, en este barrio se encontraban, además de las principales instituciones sociales, culturales y políticas judías, una miríada de redacciones de publicaciones periódicas de diferentes tamaños y frecuencias. En el caso de las editoriales idish esto es muy nítido. La mayor parte de ellas pertenecían a instituciones que se situaban dentro del Once. En el caso de las editoriales en lengua castellana, la consideración es otra pues, a excepción de la Sociedad Hebraica Argentina, los sellos más importantes eran privados. Aquí vuelve a ganar relevancia el activismo político e institucional de los editores de los sellos Israel y Candelabro. Establecerse cerca del centro de la vida institucional judía era hacer de los sellos parte de las tramas sociales y políticas judías. La ubicación de Acervo Cultural, otra de las editoriales judías en castellano de significación, en una zona de Palermo alejada del centro judío, confirma esta conjetura. Por un lado, Abraham J. Weiss, dueño del sello, situó a su empresa editorial en el mismo establecimiento de sus talleres gráficos que, no es casual en relación a su ubicación, hacía largo tiempo que no imprimían en idish; mientras que, por el otro, su activismo institucional se concentraba únicamente en la escuela judía integral Martin Buber que estaba, y aún hoy está, situada en el mismo barrio de Palermo.

Tal vez podría pensarse, en términos de hipótesis y siguiendo la idea de Bourdieu para el campo editorial francés, que la suma de estos distintos planos históricos, sociales, políticos, económicos y culturales que manifiestan diferentes formas de relación entre espacialidad y vida cultural judía, darían lugar a una dimensión sociológica dependiente de estos planos pero diferente de ellos que produciría efectos sociológicos propios, y que podríamos traducir como capital simbólico judío. Es decir, como una clase específica de capital que dotaría a las instituciones de un agregado de sentido en relación a su identificación como judías. Esto significaría, en otras palabras, que durante el período en que el Once, o bien una porción específica de este barrio, fue el centro material y simbólico de la vida colectiva judía de Buenos Aires, situar una institución en el Once suponía reforzar su carácter judío. ${ }^{27}$

Del mismo modo en que el plano muestra una clara tendencia a la concentración espacial directamente relacionada con la centralidad de este barrio dentro de la vida colectiva judía durante gran parte del siglo XX, es muy probable que si contáramos con una serie de planos

\footnotetext{
27 Esta clave analítica podría, por ejemplo, aportar un nuevo ángulo para pensar la insistencia de los dirigentes de la Sociedad Hebraica Argentina en adquirir un nuevo edificio a inicios de la década de 1930 dentro de un radio delimitado del Once, cuyo emplazamiento finalmente fue en Sarmiento 2233 y cuyo edificio fuera oficialmente inaugurado en octubre de 1943. En el mismo sentido permitiría ampliar interpretación en torno a la tensa relación que el Templo Betel situado en el barrio de Belgrano y su rabino Marshall Meyer mantuvieron con las instituciones centrales de la vida judía durante las décadas de 1960 y 1970, al mostrar que junto a las diferencias culturales y de clase entre un sector social y otro, también estaba en juego este valor simbólico específico.
} 
urbanos de los principales centros de la edición judía en el mundo, comprobaríamos que, al igual que en el caso de Buenos Aires, sería posible reducir estos centros a algunos barrios específicos. Serían por lo tanto no ya urbes como un todo, sino algunos de sus barrios los que conformarían la geografía transnacional de la edición.

\section{Conclusiones}

El punto de vista adoptado en este artículo reveló un universo social y cultural de agentes, ámbitos, intereses y relaciones, las más de las veces desatendidos por la investigación histórica, que hicieron posible y delimitaron las formas de producción y circulación del "libro judío" en Buenos Aires. El estudio de las distintas instancias que, a través de la producción, la venta o el préstamo, pusieron obras a disposición del lector de Buenos Aires, demuestra que existían numerosos y muy diversos canales a través de los que se publicaban, recibían y circulaban libros editados en diferentes centros urbanos. Estos actores, movidos por intereses comerciales, fines políticos o culturales, establecían y dinamizaban canales por los que circulaban libros, y, por ende, ideas, contribuyendo de esta suerte a la formación o bien a la ampliación del horizonte de discursos y representaciones acerca de "lo judío" en Argentina. ${ }^{28}$

Estas instancias adquieren una dimensión nueva al ser objetivadas en un plano de la ciudad. Situarlas en un espacio concreto nos permite interrogar las distintas dimensiones sociológicas que involucra esa geografía y conjeturar acerca de los modos en que un emplazamiento podía incidir o no sobre el acceso del público a los libros e incluso en la representación misma que se tenía de un sello o de una librería en particular. En tal sentido, la noción de capital simbólico judío nos ofreció una clave posible para leer la relación entre espacialidad y apuestas políticas y culturales.

La reposición de esta configuración demuestra que la disponibilidad y el consumo de la producción editorial no eran el resultado espontáneo del valor literario o ideológico intrínseco de las obras, sino que sus posibilidades y límites de existencia, desarrollo y alcance, e incluso su valor literario o ideológico, estaban en gran medida determinados por estos actores, tramas y espacios. Visibilizándose con ello el papel decisivo que desempeñó este conjunto de mediadores en la recreación y modelación de la cultura judía argentina durante gran parte del siglo veinte.

28 Alejandro Dujovne, 2010, Op. Cit.

\section{Bibliografía}

Devoto, F. 2009. Historia de la inmigración en la Argentina, Sudamericana, Buenos Aires

Dujovne, A. 2008. Cartografía de las publicaciones periódicas judías de izquierda en Argentina, 1900-1953. Revista del Museo de Antropología 1(1): 121-138.

Dujovne, A. 2010. Impresiones del judaísmo. Una sociología histórica de la producción y circulación transnacional del libro en el colectivo social judío de Buenos Aires, 1919-1979, tesis doctoral inédita, UNGSIDES, Buenos Aires

Dujovne, A. 2011. Los libros que no deben faltar en ningún hogar judío. La traducción como política cultural, 1919-1938, en Kahan, E., Setton, D., Shenquer, L. y Dujovne, A. (Comps.), Nuevos estudios sobre la vida judía en Argentina, Ediciones Lumiere, Buenos Aires, Págs. 239-261.

Bourdieu, P. 2000. Una revolución conservadora en la edición, en Intelectuales, política y poder, Eudeba, Buenos Aires

Gerchunoff, P. y P. Aguirre. 2006. La economía argentina entre la gran guerra y la gran depresión, Serie estudios y perspectivas, Cepal, Buenos Aires

Gutiérrez, L. H. y L. A. Romero. 1995. Sectores populares, cultura y política: Buenos Aires en la entreguerra, Buenos Aires, Sudamericana. Capítulo: "Sociedades barriales, bibliotecas Populares y cultura de los sectores populares: Buenos Aires, 1920-1945")

Mendelsohn, E. 1984. Jewish Politics in East Central Europe Between the World Wars, Beiner-Citrin Memorial Lecture, Harvard University, Cambridge, Mass.

Sarlo, B. 2004. El imperio de los sentimientos, Grupo Editorial Norma, Buenos Aires

Senkman, L. 2007. Ser judío en Argentina: las transformaciones de la identidad nacional, en MendesFlohr, Paul, Assis, Yom Tov y Senkman, Leonardo (Comps.), Identidades judías, modernidad y globalización, Lilmod, Buenos Aires, Págs. 403-454.

Sofer, E. 1982, From Pale to Pampa: A social history of the Jews of Buenos Aires, Holmes and Meier, Nueva York. 
